\title{
Base molecular dos fatores de virulência de Streptococcus equi
}

\author{
[Molecular basis of the virulence factors of Streptococcus equi]
}

\section{"Revisão/Review"}

\author{
Tania Alexandra Ortega Sierra ${ }^{1,2 *}$, Atzel Candido Acosta Abad ${ }^{2}$, José Wilton Pinheiro Junior ${ }^{1,2}$
}

\author{
${ }^{1}$ Programa de Pós-Graduação em Ciência Animal Tropical, Departamento de Morfologia e Fisiologia Animal, \\ Universidade Federal Rural de Pernambuco, Recife-PE, Brasil. \\ ${ }^{2}$ Laboratório de Doenças Infecciosas, Universidade Federal Rural de Pernambuco, Recife-PE, Brasil. \\ *Autor para correspondência/Corresponding author: E-mail: tanialex171@ @otmail.com
}

\section{Resumo}

Streptococcus equi subespécie equi (S. equi) é responsável pela adenite equina, doença infecto-contagiosa que ocorre com elevadas prevalências em todo o mundo. Desde a primeira descrição desta enfermidade não tem sido possível realizar o controle da mesma, o que pode ser explicado pela variabilidade nos genes de virulência. Ao longo dos anos alguns estudos foram realizados para identificar os fatores de virulência envolvidos na patogenia da enfermidade, no entanto, ainda existem algumas lacunas sobre a ocorrência e mecanismos desses genes na patogenia da doença. Desta forma, objetiva-se com esta revisão discorrer sobre os principais fatores de virulência de $S$. equi subespécie equi, os genes que participam na expressão dos mesmos e avanços no desenvolvimento de vacinas.

Palavras-chave: adenite equina; genes; fatores de virulência; vacinas.

\begin{abstract}
Streptococcus equi subspecies equi causes strangles, an infectious disease that occurs with a high prevalence worldwide. Since the first description of this disease, it has not been possible to control it, which can be explained by the variability of the virulence genes. Over the years, some studies have been carried out to identify the virulence factors involved in the pathogenesis of the disease; however, there are still some gaps regarding the occurrence and mechanisms of these genes. Therefore, the objective of this review is to discuss the main virulence factors of $S$. equi subspecies equi, the genes that participate in their expression and advances in the development of vaccines.
\end{abstract}

Keywords: strangle; genes; virulence factors; vacines.

\section{Introdução}

A adenite equina é uma doença infectocontagiosa que afeta os equinos em todo o mundo (Timoney, 2004; Stanchi et al., 2007; Libardoni et al., 2016), é preocupante do ponto de vista da saúde animal por apresentar elevada prevalência e ocasionar elevadas perdas econômicas nos rebanhos equinos (Harrington et al., 2002; Javed et al., 2016).

Esta enfermidade é ocasionada por Streptococcus equi subespécie equi (Streptococcus equi), bactéria $\beta$ hemolítica, pertence ao grupo $\mathrm{C}$ de Lancefield (Mallicote, 2015), sendo observada ao microscópio como coco Gram positivo formando cadeias ou agrupados em pares, anaeróbio facultativo, mede de 0,5 a $2 \mu \mathrm{m}$ de diâmetro, não forma esporos e é imóvel (Stanchi et al., 2007; Waller et al., 2014).

Considera-se que Streptococcus equi subespécie equi evoluiu de Streptococcus equi subespécie zooepidemicus, devido à sua semelhança no genoma (Timoney, 2004; Waller et al., 2014). No entanto, Holden et al. (2009) ao sequenciar a cepa de $S$. equi $\mathrm{Se} 4047$ determinaram que $S$. equi apresentava uma sequência mais longa que $S$. zooepidemicus, isso ocorreu porque $S$. equi adquiriu vários elementos genéticos móveis ou 
transposons, constituindo assim 16,4\% do genoma que pode carregar determinantes de virulência que estão ausentes em $S$. zooepidemicus.

A patogenia de $S$. equi não está bem elucidada, embora alguns fatores de virulência já foram descritos, os quais são difíceis de classificar em categorias específicas porque a maioria apresentam várias funções (Moraes et al., 2009), mas Slater (2007) considera quatro grupos: parede celular (peptidoglicano); cápsula de ácido hialurônico; proteínas de superfície (proteínas de união à matriz extracelular; proteínas de união ao anticorpo, proteína M) e exotoxinas (hialuronidase, estreptolisine, estreptoquinase e superantígenos), sendo os mais importantes a cápsula de ácido hialurônico e proteína M (Timoney, 2004).

\section{Parede Celular e Cápsula de Ácido Hialurônico}

Streptococcus equi apresenta um cromossomo circular completo de 2.253.793pb dos quais 2.137 são sequências de codificação, o qual foi determinado por Holden et al. (2009) a partir do sequenciamento completo de Streptococcus equi cepa 4047 (Se4047), a mesma codifica 29 proteínas relacionadas com a parede celular, pelas sequências de codificação $s r t A$ e $s r t C$.

Streptococcus equi é capsulado o que dá uma aparência de gota de mel em ágar sangue (Harrington et al., 2002). O ácido hialurônico é um polissacarídeo linear de alto peso molecular composto por $\mathrm{N}$-acetilglucosamina e ácido glucurônico (Armstrong e Johns, 1997; Slater, 2007). A cápsula de ácido hialurônico diminui a atividade fagocítica dos neutrófilos e é necessária para a atividade das proteases, das toxinas e da proteína $\mathrm{SeM}$, este ácido protege a bactéria do sistema imune do hospedeiro, impedindo que o C3b seja depositado na superfície bacteriana, interferindo na opsonização (Wibawan et al., 1999; Timoney et al., 2006; Slater, 2007).

Os operones denominados has codificam as enzimas que estão envolvidas na produção dos precursores do ácido hialurônico as quais são denominadas ácido hialurônico sintetase, em Streptococcus equi pode ser encontrado dois operones hasA, hasB, hasC e hasC, gmlU, pgi (Blank et al., 2008). A superexpressão dos operones has aumenta a produção de ácido hialurônico, mudanças em qualquer destes operones produz a diminuição de ácido hialurônico, portanto diminui a virulência da bactéria, motivo pelo qual tem sido um objetivo de estudo para a elaboração de vacinas (Anzai et al., 1999; Timoney et al., 2004; Zakeri et al., 2017).

Existem enzimas que degradam o ácido hialurônico facilitando assim a invasão realizada pela bactéria e a liberação das toxinas produzidas por ela, uma vez que melhora a adesão às células do hospedeiro e a colonização, estas são denominadas como hialuronato liasa $(H y l)$, que é codificada por SEQ2045, sequência que Se4047 adquiriu de um profago, as proteínas SEQ2045 têm tríplice cadeia de $\beta$-hélice (Baker et al., 2002; Smith et al., 2005; Lindsay et al., 2008; Holden et al., 2009), sendo enzimas codificadas por um fago que possuem menor atividade, desta forma a infecção por Streptococcus equi em alguns casos é disseminada (Holden et al., 2009); porém, Hynes e Walton (2000) indicaram que a $H y l$ pode ser um dos componentes principais para a disseminação do microrganismo a partir do sítio primário da infecção. A diminuição na expressão do gene $H y l$ aumenta os níveis de ácido hialurônico e consequentemente aumenta a resistência da bactéria à fagocitose, por outro lado reduz a adesão bacteriana às células (Timoney et al., 2006; Holden et al., 2009).

As lipoproteínas da parede celular são importantes para a sobrevivência da bactéria e estão envolvidas na absorção de nutrientes, na divisão e maturação celular, e na adesão às células do hospedeiro; também intervêm na virulência bacteriana, na retenção das proteínas externas e acredita-se que têm um papel relevante nas interações da bactéria com o hospedeiro. Destacase a ação de três lipoproteínas de Streptococcus equi, LppC (lipoproteína fosfatasse), MBL (lipoproteínas de união a metais) e HAP (proteína associada ao hialuronato), entre $1 \%$ a $3 \%$ do genoma da bactéria codifica lipoproteínas (Hamilton et al., 2006; Babu et al., 2006; Nakayama et al., 2012).

Pesquisas realizadas demonstram que o gene mbl de Streptococcus equi codifica as MBL, tem uma homologia de 72 a $92 \%$ com MtsA de Streptococcus pyogenes, o qual é uma fase de leitura aberta que codifica proteínas típicas do sistema transportador ABC (ATP binding cassette). As proteínas que compreendem este sistema transportador $\mathrm{ABC}$ são: proteína hidrófoga de membrana, proteína de união a nucleotídeos e lipoproteínas. Este sistema está relacionado com a absorção de nutrientes por parte da bactéria dentro do hospedeiro e com a produção de exotoxinas que 
interferem com a atividade dos leucócitos (Janulczyk et al., 1999; Harrington et al., 2000; Slater, 2007).

\section{Proteínas de Superfície}

A superfície celular está coberta por várias proteínas, as quais são estruturas fibrilares e considera-se que oferecem a capacidade de resistir à fagocitose (Meehan et al., 2001; Slater, 2007). As proteínas de superfície bacteriana inicialmente interagem com o hospedeiro pela adesão às células do mesmo, produzindo a estimulação da resposta imune iniciando a infecção (Tiouajni et al., 2014). As moléculas da matriz extracelular são as adesinas adequadas para as bactérias porque são abundantes no hospedeiro, entre estas se destaca o fibrinogênio e a fibronectina, as bactérias utilizam as proteínas da união a matriz extracelular denominadas proteínas ligadoras do fibrinogênio $(\mathrm{FgBP})$ e proteínas ligadoras da fibronectina (FNE) (Harrington et al., 2002).

As FgBP participam na ação antifagocítica limitando a deposição do $\mathrm{C} 3 \mathrm{~b}$ na superfície bacteriana, portanto inibem a opsonização, a fase de leitura aberta $f b p$ codifica 534 aminoácidos das proteínas ligadoras do fibrinogênio, e tem uma estrutura e sequência similar às proteínas da parede celular de outros estreptococos (Meehan et al., 1998; Meehan et al., 2000; Meehan et al., 2001). Meehan et al. (2000) comprovaram que as FgBP além de se ligar ao fibrinogênio, também são capazes de se ligar às $\mathrm{IgG}$ do equino por um mecanismo não imune, pela região FC (fragment crystallizable region) dos anticorpos (Slater, 2007), o que foi corroborado por Lewis et al. (2008) ao demonstrarem que as FgBP de $S$. equi ligam-se às IgG4 e IgG7 do equino.

A proteína $\mathrm{M}$ (SEM) é a principal FgBP e um dos principais fatores de virulência de $S$. equi associado a cápsula de ácido hialurônico, sendo a proteína SEM similar à FgBP (Meehan et al., 1998; Timoney, 2004). Boschwitz e Timoney (1994) sugerem que a proteína $\mathrm{M}$ aumenta a capacidade de $S$. equi de sobreviver no interior dos macrófagos, devido à atividade antifagocítica produzida por esta proteína, e propõem que a mesma é capaz de bloquear a deposição do C3 pela ligação com as proteínas plasmáticas. A resposta imune do animal é produzida pela presença da proteína $\mathrm{M}$, e tem sido a estrutura alvo para a produção de vacinas (Flock et al., 2006)

S. equi tem pelo menos três genes que codificam as proteínas ligadoras da fibronectina que se encontra no locus genético FCT (fibronectina e proteínas de união ao colágeno e antígeno T) (Bessen e Kalia, 2002; Tiouajni et al., 2014), as principais são FNE e SFS (Lidén et al., 2006; Holden et al., 2009). A sequência SEQ0375 (fne) codifica as FNE que são capazes de se ligarem não unicamente à fibronectina, mas também ao colágeno, melhorando assim o potencial de $S$. equi de produzir a infecção (Lindmark et al., 2001; Holden et al., 2009).

O gene fne apresenta uma deleção em uma base na metade do gene expressando-se somente um segmento do mesmo pela formação de um códon de parada na fase de leitura aberta (Harrington et al., 2000), esta deleção é conservada em todos os isolados $S$. equi analisados, resultando na perda da âncora de superfície LPXTG e leva à produção de um produto secretado, o FNE, que liga-se a fibronectina e colágeno o que provoca um incremento na virulência de $S$. equi (Lindmark et al., 2001; Holden et al., 2009). O gene sfs codifica a proteína de 40kda SFS que difere da sequência de aminoácidos de FNE (Lindmark e Guss, 1999), SFS é uma proteína de ligação da fibronectina de cepas patogénicas de $S$. equi, e pode inibir a ligação da fibronectina com o colágeno, uma vez que as ligações de SFS e do colágeno são similares (Lindmark e Guss, 1999; Ma et al., 2015).

\section{Exotoxinas}

S. equi produz várias exotoxinas que interferem com a atividade leucocítica do equino, além da nutrição bacteriana, entre as toxinas que $S$. equi produzem encontra-se a hialuronidase, estreptolisina, estreptoquinase, superantígenos (Slater, 2007; Holden et al., 2009).

As estreptolisinas $\mathrm{S}$ são toxinas extracelulares citolíticas, que produzem a lises dos PMN (polimorfonucleares) e dos macrófagos mediante ação tóxica sobre as membranas mitocondriais, além desta atividade antifagocítica, também estão envolvidas na nutrição bacteriana a partir da produção de nutrientes elaborados pela lise celular e capacidade $\beta$-hemolítica (Flanagan et al., 1998; Harrington et al., 2002; Slater, 2007). De acordo com o estudo de Waller et al. (2011) a estreptolisina é codificada por SagA na cepa 4047 de S. equi, o qual Humar et al. (2002) afirmam que é um operon de nove genes similar ao dos estreptococos do grupo A.

A estreptoquinase é um ativador do plasminógeno, isto é, transforma plasminogênio em plasmina, o que permite atravessar as 
membranas e contribui com a invasão do microrganismo nas células do hospedeiro, sugerindo que a região $s k c$ codifica a produção de estreptoquinase e difere geneticamente das proteínas elaboradas pelo ser humano (Tewodros et al., 1996; Malke et al., 2000).

As exotoxinas pirogênicas ou superantígenos associados aos fagos de $S$. equi são quatro: SeeI, SeeH, SeeL e SeeM, e são codificadas pelos genes seeI, seeH, seeL e seeM, que encontram-se nos profagos $\varphi$ SEQ3 (seeL e seeM) e $\varphi$ SEQ4 (seeI e seeH), seeI, seeH, seeL e seeM têm uma homologia de $96 \%$ a $99 \%$ com os genes codificadores de superantígenos produzidos por $S$. pyogenes (Holden et al., 2009).

Os superantígenos são moléculas imune estimuladoras altamente potentes, induzem uma proliferação linfocítica descontrolada e apresentam atividades mitogênicas e pirogênicas (Paillot et al., 2012; Commons et al., 2014). Holden et al. (2009) demonstraram que os genes que codificam SeeM e SeeL não são encontrados exclusivamente em cepas de S. equi, também podem ser detectadas no genoma de $S$. zooepidemicus, no entanto, os genes que codificam SeeI e SeeH são exclusivos de S. equi, e por isso são utilizados para a diferenciação molecular das espécies de Streptococcus equi (Artiushin et al., 2002). Velineni et al. (2017) identificaram o gene seeI em isolados de $S$. zooepidemicus, o que indica a similaridade genômica entre as duas espécies e a impossibilidade de continuar usando o gene seeI no diagnóstico molecular do Streptococcus equi subspecies equi.

\section{Avanços na Produção de Vacinas}

A prevenção da Adenite Equina pode ser realizada com a utilização de vacinas. Atualmente são comercializadas vacinas vivas modificadas baseadas na proteína $\mathrm{M}$, que foram elaboradas com o objetivo de estimular a imunidade humoral (Waller et al., 2014) e produz uma boa resposta imunológica nos três meses pós-vacinação (Slater, 2007). Por outro lado, este tipo de vacinas apresentaram algumas desvantagens, entre as quais estão os efeitos adversos como a apresentação de sinais clínicos, que pode ocorrer principalmente em animais com idade inferior a um ano (Borst et al., 2011; Cursons et al., 2015), reação inflamatória local e formação de abscessos no sitio de aplicação quando a mesma é intramuscular (Timoney, 2004; Boyle et al., 2018) e resultados falsos positivos nas provas convencionais como ELISA, provas microbiológicas e PCR (Waller, 2013).

Timoney et al. (2007) concluíram que a imunidade deve ser estimulada ao nível das mucosas nasofaríngeas no momento da aplicação, porque foi demonstrado que os animais convalescentes apresentam títulos altos de anticorpos nas mucosas, em contrapartida a titulação de anticorpos no soro foi baixa. Foram realizados alguns estudos com a produção de vacinas sem a proteína $\mathrm{M}$ ou a cápsula de ácido hialurônico, Flock et al. (2004) realizaram a imunização de camundongos com proteínas recombinantes EAG (proteína de união à globulina, albumina e imunoglobulina G), SFS e FNZ, com o veículo EtxB, e concluíram que a combinação entre EAG e FNZ com EtxB pode ser adequada para a elaboração de vacinas eficientes e inócuas. Em outro estudo, Flock et al. (2006) utilizaram as proteínas recombinantes EAG, CNE (proteína de união ao colágeno), SclC (uma proteína similar ao colágeno) e FNBE (proteína de união à fibronecina), e observaram que a sinergia entre EAG e CNE produzem uma elevada imunidade em camundongos em comparação com o resto das proteínas utilizadas nesta investigação, enquanto a FNBE não estimulou imunidade alguma.

Robinson et al. (2015) produziram uma vacina com a deleção de seis genes entre os quais estão sagA, hasA e $s e M$, e obtiveram um bom resultado porque conseguiram eliminar a característica de resistência à fagocitose da bactéria, e também em ausência de $s a g A$ a mesma não pode produzir SFS. Outras pesquisas não obtiveram bons resultados de proteção, porque não produzem imunidade suficiente para poder resistir a uma infecção natural, além de produzirem reações adversas (Timoney e Mukhtar, 1993; Timoney, 2004; Slater, 2007).

$\mathrm{Na}$ atualidade, uma nova vacina recombinante foi testada com várias proteínas de $S$. equi, e bons resultados foram obtidos com aumento da eficácia da vacina frente à infecção natural e sem efeitos adversos significativos. Entretanto, a análise de custo-beneficio deve ser realizada e para isso novas pesquisas devem ser conduzidas (Robinson et al., 2018).

\section{Considerações finais}

Apesar dos avanços nas pesquisas com genes de virulência de $S$. equi subespécie equi ainda não é possível elucidar todas as lacunas na 
patogênese da adenite equina. A realização de estudos sobre os fatores de virulência produzidos por S. equi subsp. equi são importantes do ponto de vista da saúde animal e biotecnológico.

\section{Conflito de Interesse}

Os autores declaram não existir conflito de interesse.

\section{Agradecimentos}

Os autores agradecem à Coordenação de Aperfeiçoamento de Pessoal de Ensino Superior (CAPES).

\section{Referências}

Anzai, T.; Timoney, J.; Kuwamoto, Y.; Fujita, Y.; Wada, R.; Inoue, T. In vivo pathogenicity and resistance to phagocytosis of Streptococcus equi strains with different levels of capsule expression. Veterinary Microbiology, 67: 277-286, 1999.

Armstrong, D.; Johns, M. Culture conditions affect the molecular weight properties of hyaluronic acid produced by Streptococcus zooepidemicus. Applied and Environmental Microbiology, 63(7), 2759-2764, 1997.

Artiushin, S.; Timoney, J.; Sheoran, A.; Muthupalani, S. Characterization and immunogenicity of pyrogenic mitogens SePE$\mathrm{H}$ and SePE-I of Streptococcus equi. Microbial Pathogenesis, 32: 71-85, 2002.

Babu, M.; Priya, M.; Selvan, A.; Madera, M.; Gough, J.; Aravind, L.; Sankaran, K. A Database of Bacterial Lipoproteins (DOLOP) with Functional assignments to predicted lipoproteins. Journal of Bacteriology, 188(8): 2761-2773, 2006.

Baker, J.; Dong, S.; Pritchard, D. The hyaluronan lyase of Streptococcus pyogenes bacteriophage H4489A. Biochemical Journal, 365: 317-322, 2002.

Bessen, D.; Kalia, A. Genomic localization of a t serotype locus to a recombinatorial zone encoding extracellular matrix-binding proteins in Streptococcus pyogenes. Infection and Immunity, 70(3); 1159-1167, 2002.

Blank, L.; Hugenholtz, P.; Nielsen, L. Evolution of the hyaluronic acid synthesis (has) operon in Streptococcus zooepidemicus and other pathogenic Streptococci. Journal of Molecular Evolution, doi:10.1007/s00239-008-9117-1, 2008.
Borst, L.; Patterson, S.; Lanka, S.; Barger, A.; Fredrickson, R.; Maddox, C. Evaluation of a commercially available modified live Streptococcus equi subsp equi vaccine in ponies. American Journal of Veterinary Research, 72(8): 1130-1138, 2011.

Boschwitz, J.; Timoney, J. Inhibition of C3 Deposition on Streptococcus equi subsp. equi by M Protein: a Mechanism for Survival in Equine Blood. Infection and Immunity, 62(8): 3515-3520, 1994.

Boyle, A.; Timoney, J.; Newton, J.; Hines, M.; Waller, A.; Buchanan, B. Streptococcus equi infections in horses: guidelines for treatment, control, and prevention of strangles-revised consensus statement. Journal of Veterinary Internal Medicine, 32; 633-647, 2018.

Commons, R.; Smeesters, P.; Proft, T.; Fraser, J.; Robins-Browne, R.; Curtis, N. Streptococcal superantigens: categorization and clinical associations. Trends in Molecular Medicine, 20(1): 48-62, 2014.

Cursons, R.; Patty, O.; Steward, K.; Waller, A. Strangles in horses can be caused by vaccination with Pinnacle I. N. Vaccine, 33: 3440-3443, 2015.

Flanagan, J.; Collin, N.; Timoney, J.; Mitchell, T.; Mumford, J.; Chanter, N. Characterization of the haemolytic activity of Streptococcus equi. Microbial Pathogenesis, 24: 211-221, 1998.

Flock, M.; Jacobsson, K.; Frykberg, L.; Hirst, T.; Franklin, A.; Guss, B.; Flock, J. Recombinant Streptococcus equi proteins protect mice in challenge experiments and induce immune response in horses. Infection and Immunity, 72(6): 3228-3236, 2004.

Flock, M.; Karlström, A.; Lannergård, J.; Guss, B.; Flock, J.I. Protective effect of vaccination with recombinant proteins from Streptococcus equi subspecies equi in a strangles model in the mouse. Vaccine, 24: 4144-4151, 2006.

Hamilton, A.; Robinson, C.; Sutcliffe, I.; Slater, J.; Maskell, D.; Davis-Poynter, N.; Smith, K.; Waller, A.; Harrington, D. mutation of the maturase lipoprotein attenuates the virulence of Streptococcus equi to a greater extent than does loss of general lipoprotein lipidation. Infection and Immunity, 74(12): 6907-6919, 2006.

Harrington, D.; Greated, J.; Chanter, N.; Sutcliffe, I. Identification of lipoprotein homologues of Pneumococcal PsaA in the equine pathogens Streptococcus equi and Streptococcus 
zooepidemicus. Infection and Immunity, 68(10): 6048-6051, 2000.

Harrington, D.; Sutcliffe, I.; Chanter, N. The molecular basis of Streptococcus equi infection and disease. Microbes and Infection, 4: 501510, 2002.

Holden, M.; Heather, Z.; Paillot, R.; Steward, K.; Webb, K.; Ainslie, F.; Jourdan, T.; Bason, N.; Holroyd, N.; Mungall, K.; Quail, M.; Sanders, M.; Simmonds, M.; Willey, D.; Brooks, K.; Aanensen, D.; Spratt, B.; Jolley, K.; Maiden, M.; Kehoe, M.; Chanter, N.; Bentley, S.; Robinson, C.; Maskell, D.; Parkhill, J.; Waller, A. Genomic evidence for the evolution of Streptococcus equi: host restriction, increased virulence, and genetic exchange with human pathogens. PLoS Pathogens, 5(3): e1000346, 2009.

Humar, D.; Datta, V.; Bast, D.; Beall, B.; De Azavedo, J.; Nizet, V. Streptolysin S and necrotising infections produced by group $\mathrm{G}$ streptococcus. The Lancet, 359: 124-129, 2002.

Hynes, W.; Walton, S. Hyaluronidases of Grampositive bacteria. FEMS Microbiology Letters, 183: 201-207, 2000.

Janulczyk, R.; Pallon, J.; Björk, L. Identification and characterization of a Streptococcus pyogenes $\mathrm{ABC}$ transporter with multiple specificity for metal cations. Molecular Microbiology, 34(3): 596-606, 1999.

Javed, R.; Taku, A.; Gangil, R.; Sharma, R. Molecular characterization of virulence genes of Streptococcus equi subsp. equi and Streptococcus equi subsp. zooepidemicus in equines. Veterinary World, 9(8): 875-881, 2016.

Libardoni, F.; Machado, G.; Gressler, L.T.; Kowalski, A.P.; Diehl, G.N.; Carboneiro dos Santos, L.; Corbellini, L.G.; Castagna de Vargas, A. Prevalence of Streptococcus equi subsp. equi in horses and associated risk factors in the State of Rio Grande do Sul, Brazil. Research in Veterinary Science, 104: 53-57, 2016.

Lidén, Å; Karlström, Å.; Lannergård, J.; Kalamajski, S.; Guss, B.; Rubin, K.; Rydén, C. A fibronectin-binding protein from Streptococcus equi binds collagen and modulates cell-mediated collagen gel contraction. Biochemical and Biophysical Research Communications, 340: 604-610, 2006.
Lindmark, H.; Guss, B. SFS, a Novel fibronectinbinding protein from streptococcus equi, inhibits the binding between fibronectin and collagen. Infection and Immunity, 67(5), 2383-2388, 1999.

Lindmark, H.; Nilsson, M.; Guss, B. Comparison of the Fibronectin-Binding Protein FNE from Streptococcus equi subspecies equi with FNZ from $S$. equi subspecies zooepidemicus reveals a major and conserved difference. Infection and Immunity, 69(5): 3159-3163, 2001.

Lindsay, A.M.; Zhang, M.; Mitchell, Z.; Holden, M.; Waller, A.; Sutcliffe, I.; Black, G. The Streptococcus equi prophage-encoded protein SEQ2045 is a hyaluronan-specific hyaluronate lyase that is produced during equine infection.

Microbiology, 155: 443-449, 2008.

Ma, W.; Ma, H.; Fogerty, F.; Mosher, D. Bivalent ligation of the collagen-binding modules of fibronectin by SFS, a non-anchored bacterial protein of Streptococcus equi. The Journal of Biological Chemestry, 290(8): 4866-4876, 2015.

Malke, H.; Steiner, K.; Gase, K.; Frank, C. Expression and regulation of the streptokinase gene. Methods, 21: 111-124, 2000.

Mallicote, M. Update on Streptococcus equi subsp equi infections. Veterinary Clinics of North America: Equine Practice. 31: 27-41, 2015.

Meehan, M.; Lynagh, Y.; Woods, C.; Owen, P. The fibrinogen-binding protein (FgBP) of Streptococcus equi subsp. equi additionally binds $\operatorname{IgG}$ and contributes to virulence in a mouse model. Microbiology, 147: 3311-3322, 2001.

Meehan, M.; Muldowney, D.; Watkins, N.; Owen, $\mathrm{P}$. Localization and characterization of the ligand-binding domain of the fibrinogenbinding protein (FgBP) of Streptococcus equi subsp. equi. Microbiology, 146: 1187-1194, 2000.

Meehan, M.; Nowlan, P.; Owen, P. Affinity purification and characterization of a fibrinogen-binding protein complex which protects mice against lethal challenge with Streptococcus equi subsp. equi. Microbiology, 144: 993-1003, 1998.

Moraes, C. M.; Vargas, A. P.; Leite, F. P.; Nogueira, C. E.; Turnes, C. G. Adenite equina: sua etiologia, diagnóstico e controle. Ciência Rural, Santa Maria, 39(6): 1944-1952, 2009.

Nakayama, H.; Kurokawa, K.; Lee, B. Lipoproteins in bacteria: structures and 
biosynthetic pathways. The FEBS Journal, 4247-4268, 2012.

Paillot, R.; Wright, N.; McLean, R.; Darby, A.; Anderson, E.; Steward, K.; Webb, K.; Priestnall, S.; Erles, K.; Smith, K.; Efstratiou, A.; Holden, M.; Robinson, C.; Waller, A. Diversity of superantigens in Streptococcus equi and zooepidemicus populations. Journal of Equine Veterinary Science, 32: S24-S25, 2012.

Robinson, C.; Frykberg, L.; Flock, M.; Guss, B.; Waller, A.; Flock, J.I. Strangvac: A recombinant fusion protein vaccine that protects against strangles, caused by Streptococcus equi. Vaccine, 36: 1484-1490, 2018.

Robinson, C.; Heather, Z.; Slater, J.; Potts, N.; Steward, K.; Maskell, D.; Fontaine, M.; Lee, J.; Smith, K.; Waller, A. Vaccination with a live multi-gene deletion strain protects horses against virulent challenge with Streptococcus equi. Vaccine, 33: 1160-1167, 2015.

Slater, J. Bacterial Infections of the Equine Respiratory Tract. In: B. McGorum, P.D.; E. Robinson, E.; Schumacher, J. Equine Respiratory Medicine and Surgery. Philadelphia: Elsevier, 2007. p.314-324

Smith, N.; Taylor, E.; Lindsay, A.M.; Charnock, S.; Turkenburg, J.; Dodson, E.; Davies, G.; Black, G. Structure of a group A streptococcal phage-encoded virulence factor reveals a catalytically active triple-stranded b-helix. Proceedings of the National Academy of Sciences, 102(49): 17652-17657, 2005.

Stanchi, N.O.; Martino, P.; Gentilini, E.; Reinoso, E.; Echeverría, M.; Leardini, N.; Copes, J. Microbiología veterinaria. Buenos Aires: Inter-médica, 2007.

Tewodros, W.; Karlsson, I.; Kronvall, G. Allelic variation of the streptokinase gene in Bhemolytic streptococci group $\mathrm{C}$ and $\mathrm{G}$ isolates of human origin. FEMS Immunology and Medical Microbiology, 13: 29-34, 1996.

Timoney, J. The pathogenic equine streptococci. Veterinary Research, a Journal on Animal Infection, 35(4): 397-409, 2004.

Timoney, J.; Mukhtar, M. The protective M proteins of the equine group $\mathrm{C}$ streptococci. Veterinary Microbiology, 3(4): 389-395, 1993.
Timoney, J.; Kumar, P.; Muthupalani, S. Interaction of Streptococcus equi with the equine nasopharynx. International Congress Series, 1289: 267-270, 2006.

Timoney, J.; Qin, A.; Muthupalani, S.; Artiushin, S. Vaccine potential of novel surface exposed and secreted proteins of Streptococcus equi. Vaccine, 25: 5583-5590, 2007.

Tiouajni, M.; Durand, D.; Blondeau, K.; Graille, M.; Urvoas, A.; Valerio-Lepiniec, M.; Guellouz, A.; Aumont-Nicaise, M.; Minard, P.; Tilbeurgh, H. Structural and functional analysis of the fibronectin-binding protein FNE from Streptococcus equi spp. equi. The FEBS Journal, 281: 5513-5531, 2014.

Velineni, S.; Sheoran, A.; Breathnach, C.; Rash, N.; Paillot, R.; Timoney, J. Mitogenic Equine Isolates of Streptococcus zooepidemicus From North America Host Superantigen Genes Similar to Those Hosted in Europe. Journal of Equine Veterinary Science, 49: 40-44, 2017.

Waller, A. Strangles: Taking steps towards eradication. Veterinary Microbiology, 167: 50-60, 2013.

Waller, A.S.; Sellon, D.C.; Sweeney, C.R.; Timoney, P.J.; Newton, R.; Hines, M.T. Streptococcal Infections. In Sellon, D.; Long, M. Equine Infectious Diseases. United States: Elsevier, 2014.p.265-277.

Waller, A.; Paillot, R; Timoney, J. Streptococcus equi: a pathogen restricted to one host. Journal of Medical Microbiology, 60: 1231-1240, 2011.

Wibawan, W.; Pasaribu, F.; Utama, I.; Abdulmawjood, A.; Lämmler, C. The role of hyaluronic acid capsular material of Streptococcus equi subsp. zooepidemicus in mediating adherence to HeLa cells and in resisting phagocytosis. Research in Veterinary Science, doi:10.1053/rvsc.1998.0287, 1999.

Zakeri, A.; Rasaee, M.; Pourzardosht, N. Enhanced hyluronic acid production in Streptococcus zooepidemicus by over expressing HasA and molecular weight control with Niscin and glucose. Biotechnology Reports, doi:https://doi.org/10.1016/j.btre.2017.02.007, 2017. 